\title{
The Emotional Effect of Semi Classical Carnatic Music using Linguistic Aggregated Fuzzy Relational Maps
}

\author{
A. Victor Devadoss \\ Head and Associate Professor \\ $P G$ and Department of Mathematics \\ Loyola College, Chennai, India
}

\author{
S. Aseervatham \\ Research Scholar \\ $P G$ and Department of Mathematics \\ Loyola College, Chennai, India
}

\begin{abstract}
People's primary motivation for listening to music is its emotional effect. Assigning musical emotion to a song segment in a deterministic way does not work well because not all people share the same feeling for a song. The success of becoming a hit song from the same raga song among people is its musical features within the song. The subjective nature of listening knowledge of human to different Carnatic raga perception suggests that fuzzy logic is a more appropriate tool for an unsupervised data. This paper deals with the Linguistic Aggregated Fuzzy Relational Maps which is the combination of the linguistic variables with their aggregation and Fuzzy Relational Maps. LAFRMs method is mostly used for humans' expression or feelings expressed for the causes and its effects in terms of linguistic languages. The process of expressing linguistic knowledge is described as unsupervised data. The problem of musical emotions caused by thirteen Carnatic ragas based Tamil songs were analyzed using LAFRMs. This is the first attempt of using fuzzy tool to analyze this problem. The result shows the effectiveness of the proposed method for the application in this linguistic environment.
\end{abstract}

\section{Keywords}

Linguistic variables, Triangular fuzzy number, Fuzzy relational maps, Carnatic raga, Musical feature.

\section{INTRODUCTION}

The meaning of the music in terms of emotions is subjective in nature. Several psychological conditions of the Human Beings decide the emotion category of a song or musical excerpts. Representations of musical emotions with the psychology remain a dynamic topic for research. There are several computational models available for mood classification. Music is used in several applications in society that believes its effectiveness in evoking emotions. Carnatic music from Indian tradition has rasas (emotions) and its singing timing of the day. The use of Carnatic ragas in Indian movies and devotional songs are more often utilized by Indian composers. For these compositions, the emotional experiences vary from one person to another in an uncertain manner. The developed Linguistic Aggregated FRM model of Fuzzy Relational Maps is used as a major method to assign the emotional factors to the appropriate Carnatic ragas with musical features through musical excerpts. Fuzzy Relational Maps are introduced by W.B. Vasantha and Yasmin Sultana [7]. Every human being judgments on experiences are described in terms of linguistic language. Moreover instead of having numerical value approach for innermost feelings of human being, linguistic memberships are more sensible in bringing communications more clearly. Linguistic variable assessments are applied in FRMs to develop the model. These FRMs are basically derived from relating two disjoint units of domains by causal relations. The causal relations are alternated by different linguistic level of experiences of an individual or a group is observed. Further linguistic variables are aggregated with the use of triangular fuzzy numbers. Then the model has been developed to apply in an uncertain linguistic environment. Few ideas about Carnatic ragas and Musical Features are explained in this section.

Rest of this paper consists of three sections. In section 2, the preliminary definitions on the proposed method are given. The problem description and adaptation of the method discussed in section 3. Conclusions are derived in the final section to show the effectiveness of the proposed method.

\subsection{Carnatic Ragas}

One of the melodic modes used in Indian Classical music is a raga. They use a series of five or more musical notes in which a melody is constructed. These ragas are associated with seasons and emotions in the Indian musical tradition. Indian film and devotional song composers often use non-classical form ragas in their compositions. There are 72 Melakarta ragas and derived sub ragas in the system. Improvisation plays a major role in Carnatic music. Some musical feature ideas used in composition are explained below.

\subsubsection{Mode Feature}

It can be derived from various scales (ie.) families of pitches where as only thirteen Carnatic ragas structured from these scales are considered for this study.

\subsubsection{Rhythm Feature}

It is generated by divisions of the beat. Rhythm strength, regularity and tempo are closely related with people's moods or responses (Liu et al., 2003) [4]. It is the phase of time in music. Regular and irregular rhythms are mostly used in compositions.

\subsubsection{Harmony Feature}

It is the art of combining pitches into chords in which several notes played simultaneously at a time. Chord, progression, consonance, dissonance are its techniques in Western music.

\section{PROPOSED METHODOLOGY}

The basic definitions of fuzzy relational maps and the developed linguistic aggregated fuzzy relational maps are briefly explained in this section.

\subsection{Fuzzy Relational Maps (FRMs)}

A FRM is structured as a map by making causal relationships between the domain and range space elements. The elements of the domain space are taken from the real vector space of dimension $n$ and that of the range space are real vector space of dimension $m$ ( $m$ in general need not be equal to $n$ ). We denote by $D$ the set of nodes $D_{1}, \ldots, D_{n}$ of the domain space where $D_{i}=\left\{x_{1}, \ldots, x_{n}\right) / x_{i}=0$ or 1$\}$ for $i=1, \ldots, n$. If $x_{i}=1$, it means that the node $D_{i}$ is in the ON stage and if $x_{i}=0$ it means that the node $D_{i}$ is in the OFF state. Similarly $R$ denotes the set of nodes $R_{1}, \ldots, R_{m}$ of the range space, where 
$R_{j}=\left\{x_{1}, x_{2}, \ldots, x_{m}\right) / x_{j}=0$ or 1$\}$ for $j=1, \ldots, m$. If $x_{j}=1$ it means that the node $R_{j}$ is in the ON state and if $x_{j}=0$ it means that the node $R_{j}$ is in the OFF state.

\subsection{Linguistic FRM}

A directed graph or a map from $D$ to $R$ is constructed as Linguistic FRM with causes and their effects as nodes and their linguistic causalities as edges. It represents linguistic causal relationship between spaces $D$ and $R$.

\subsection{Linguistic Experience Matrices}

Let ${ }_{l} D_{i}$ and ${ }_{l} R_{j}$ denote the two nodes of Linguistic FRM. The directed edge from ${ }_{l} D_{i}$ to ${ }_{l} R_{j}$ denotes the causality relation of ${ }_{l} D_{i}$ on ${ }_{l} R_{j}$. Every edge in the Linguistic FRM is weighted with the different level of linguistic terms. Linguistic experience matrices are formed with the use of number of experience strength of group for different levels. These level matrices are denoted by $N_{\text {Level-(k)}}^{(k)}$ and defined as,

$N_{\text {Level-(k) }}^{(k)}=\left[b_{i j}^{(k)}\right] \forall k=$ low, medium, high...etc, where $b_{i j}$ is the weight of the edge $D_{i l} R_{j}$. The weight of the edge from any node ${ }_{l} D_{i}$ to any node $R_{j}$ is described by the linguistic terms $\{0$ (no effect), $v l$ (very low), $l$ (low), $m$ (medium), $h$ (high), $v h$ (very high) $\}$. According to the problem description, these linguistic term levels can be extended further.

\subsection{Linguistic Relational Matrix}

Let ${ }_{l} D_{1}, \ldots,{ }_{l} D_{n}$ be the nodes of the domain space $D$ of Linguistic FRM and ${ }_{l} R_{l}, \ldots, R_{m}$ be the nodes of the range space $R$ of an Linguistic FRM. Let the matrix $M$ be defined as

$$
M=\left[e_{i j}\right]
$$

where $e_{i j}=\max$ linguisticlevel $\left(\max \left(b_{i j}^{(1)}, b_{i j}^{(2)}, b_{i j}^{(3)}\right)\right)$, and $e_{i j}$ is the maximum linguistic weight of the directed edge ${ }_{l} D_{i}$ ${ }_{l} R_{j}$ (or ${ }_{l} R_{j l} D_{i}$ ), $M$ is called the linguistic relational matrix of the Linguistic FRM with linguistic variable entries.

\subsection{Fuzzy Nodes}

When the nodes of the Linguistic FRM are fuzzy sets then they are called fuzzy nodes. Here fuzzy sets are defined by converting linguistic terms into fuzzy numbers.

\subsection{Triangular Relational Matrix}

The matrix $M$ with the linguistic variables is converted with the entries of fuzzy numbers for the corresponding linguistic variables. Triangular fuzzy numbers $\left(a_{i j}, b_{i j}, c_{i j}\right)$ for the linguistic variables are considered. Then triangular relational matrix $\operatorname{Tr}(M)$ defined as, $\operatorname{Tr}(M)=\left[t_{i j}\right]$ where $t_{i j}=\left(a_{i j}, b_{i j}, c_{i j}\right) \quad \forall$ $i=1,2, \ldots, n \& j=1,2, \ldots, m$.

\subsection{Relational Matrix with Left and Right \\ Values}

This triangular relational matrix is aggregated by finding left $(l s)$ and right $(r s)$ values of triangular numbers. The following formulae are used to calculate this:

$$
\begin{gathered}
l s_{i j}=\frac{b_{i j}}{1+b_{i j}-a_{i j}} \& r s_{i j}=\frac{c_{i j}}{1+c_{i j}-b_{i j}} . \\
\text { Then } \operatorname{Tr}(M)=\left[\left(l s_{i j}, r s_{i j}\right)\right] .
\end{gathered}
$$

\subsection{Linguistic Aggregated Relational matrix} Then triangular relational matrix is further aggregated as normalized value to get single number. This is done by, $x_{i j}=\frac{l s_{i j}\left(1-l s_{i j}\right)+r s_{i j} \cdot r s_{i j}}{1+r s_{i j}-l s_{i j}}$. Then $L A(M)=\left[x_{i j}\right] \quad \forall i=$ $1,2, \ldots, n \& j=1,2, \ldots, m$. The entries of $L A(M)$ are called as triangular aggregated vectors.

\subsection{Instantaneous stage vector}

Let $D_{l}, \ldots,{ }_{l} D_{n}$ and ${ }_{l} R_{l}, \ldots,{ }_{l} R_{m}$ denote the nodes of the LAFRM. Let $A=\left(a_{1}, \ldots, a_{n}\right) a_{i} \in\{0, \pm 1\} . \quad A$ is called the instantaneous stage vector of the domain space and it denotes the $O N-O F F$ position of the nodes at any instant. Similarly let $B=$ $\left(b_{1}, \ldots, b_{m}\right) b_{j} \in\{0, \pm 1\} . B$ is called instantaneous stage vector of the range space and it denotes the $O N-O F F$ position of the nodes at any instant. Then $a_{i}$ or $b_{j}=0$ if $a_{i}$ or $b_{j}$ is $O F F$ and $a_{i}$ or $b_{j}=1$ if $a_{i}$ or $b_{j}$ is $O N \forall i=1,2, \ldots, n \& j=1,2, \ldots, m$.

\subsection{Cyclic and Acyclic}

The LAFRM is said to be a cyclic if its edges possess a directed cycle. An LAFRM is said to be acyclic if its edges do not possess any directed cycle.

\subsection{LAFRM with Feedback}

The LAFRM with cycles is said to be an LAFRM with feedback.

\subsection{Dynamical system}

The LAFRM is said to be a dynamical system, when the causal relations flow through a cycle in a revolutionary manner with feedback.

\subsection{Hidden Pattern}

Let ${ }_{l} D_{i l} R_{j}\left({ }_{l} R_{j l} D_{i}\right), 1 \leq i \leq n, 1 \leq j \leq m$. When ${ }_{l} R_{i}$ (or $\left.{ }_{l} D_{j}\right)$ is switched $O N$ and if causality flows through edges of the cycle and if it again causes ${ }_{l} R_{i}$ (or ${ }_{l} D_{j}$ ), the dynamical system goes round and round. This is true for any node ${ }_{l} R_{j}\left(\right.$ or $\left.{ }_{l} D_{i}\right)$ for $1 \leq j$ $\leq m$ (or $1 \leq i \leq n)$. The equilibrium stage of this dynamical system is called the hidden pattern.

\subsection{Fixed Point}

If the equilibrium state of a dynamical system is a unique stage vector, then it is called a fixed point. Consider an LAFRM with ${ }_{l} R_{l}, R_{2}, \ldots,{ }_{l} R_{m}$ and ${ }_{l} D_{l}, D_{2}, \ldots l D_{n}$ as nodes. For example, let us start the dynamical system by switching on ${ }_{l} R_{l}$ (or $D_{l}$ ). Let us assume that the LAFRM settles down with ${ }_{l} R_{l}$ and ${ }_{l} R_{m}$ (or ${ }_{l} D_{l}$ and ${ }_{l} D_{n}$ ) i.e. the stage vector remains as $(1$, $0, \ldots, 0,1)$ in $R$ (or $1,0,0, \ldots, 0,1)$ in $D$ ). This state vector is called the fixed point.

\subsection{Limit Cycle}

If the LAFRM settles down with a state vector repeating in the form $\quad A_{I} \rightarrow A_{2} \rightarrow A_{3} \rightarrow \ldots \rightarrow A_{i} \rightarrow A_{I} \quad$ (or $\quad B_{I} \rightarrow \quad B_{2} \rightarrow$ $\ldots \rightarrow B_{j} \rightarrow B_{l}$ ) then this equilibrium state is called a limit cycle.

\subsection{Algorithmic procedure of finding Hidden pattern}

Step 1: Let $L A(M)$ be the dynamical system matrix formed by the linguistic relationship between the nodes ${ }_{l} D_{i}$ and ${ }_{l} R_{j} \quad \forall i=$ $1,2, \ldots, n \& j=1,2, \ldots, m$.

Step 2: Let us find the hidden pattern when ${ }_{l} D_{l}$ is switched $\mathrm{ON}$. This is done by fixing the state vector $X_{I}=(1,0,0, \ldots, 0)$ (instead of ${ }_{l} D_{l}=(1,0,0, \ldots, 0)$ ). When an input is given as the vector $X_{l}$, the data should pass through the relation matrix $L A(M)$ by multiplying $X_{I}$ with $L A(M)$.

Step 3: Let $X_{1} L A(M)=\left(b_{1}, b_{2}, \ldots, b_{m}\right)$ be a triangular aggregated vector. Then the threshold operation $(\hookrightarrow)$ on 
$X_{l} L A(M)$ is done by replacing $b_{j}$ by 1 if $b_{j}$ is the maximum value in $X_{l} L A(M)$, otherwise $b_{j}$ by 0 . The thresholded vector is called as $Y_{l}$. ie. $Y_{l}=\left(b_{1}, b_{2}, \ldots, b_{m}{ }^{\prime}\right)$.

Step 4: Then find $Y_{l} L A(M)^{T}$ by multiplying $Y_{l}$ with $L A(M)^{T}$. Let $Y_{l} L A(M)^{T}=\left(a_{1}, a_{2}, \ldots, a_{n}\right)$ be a triangular aggregated vector. The threshold operation is done for $Y_{l} L A(M)^{T}$ as in step 3 for the vectors $a_{i}$ 's. Now the thresholded vectors are called as $X_{2}$.

Step 5: This procedure is repeated till we get a limit cycle or a fixed point as $X_{p}=X_{q}$ and $Y_{r}=Y_{s}$ (for some $p \neq q$ and $r \neq s$ ) in both $D$ and $R$ domains respectively. In general, this procedure can be repeated for any vector ${ }_{l} D_{i} ; i=1,2, \ldots, n$.

\section{DESCRIPTION OF THE PROBLEM}

The following Carnatic raga based songs are taken for the analysis to assign emotional experiences. Thirteen familiar ragas based totally 78 popular Tamil movie songs and Christian devotional songs were chosen for the survey. For this work, 20 undergraduate students are interviewed and played those songs. Then their opinions are recorded on the emotions level sheet while listening to each raga songs.

The following attributes of ragas based songs are taken as domain space elements of LAFRM.

Each raga musical notes are given in basic $C$ tone. It will be applicable for all notes to form raga.

\section{${ }_{l} D_{1}-$ Sindhu Bhairavi}

- Its notes are C, Db, Eb, F, G, Ab, Bb, C

\section{${ }_{l} D_{2}-$ Hindolam}

- Its notes are $\mathrm{C}, \mathrm{Eb}, \mathrm{F}, \mathrm{Ab}, \mathrm{Bb}, \mathrm{C}$

\section{${ }_{1} D_{3}-$ Keeravani}

- Its notes are C, D, Eb, F, G, Ab, B, C

\section{${ }_{l} D_{4}-$ Karaharapriya}

- Its notes are C, D, Eb, F, G, A, Bb, C

\section{${ }_{1} D_{5}$ - Shiva Ranjani}

- Its notes are C, D, Eb, G, A, C

${ }_{l} D_{6}-$ Charukeshi

- Its notes are C, D, E, F, G, Ab, Bb, C

\section{${ }_{1} D_{7}$ - Mohanam}

- Its notes are C, D, E, G, A, C

\section{${ }_{1} D_{8}-$ Chalanattai}

- Its notes are C, D\#, E, F, G, A\#, B, C

\section{${ }_{l} D_{9}-$ Suba Panthuvarali}

- Its notes are C, Db, Eb, F\#, G, Ab, B, C

\section{${ }_{1} D_{10}-$ Simhendra Madhyamam}

- Its notes are C, D, Eb, F\#, G, Ab, B, C

${ }_{l} D_{11}$ - Mecha Kalyani

- Its notes are C, D, E, F\#, G, A, B, C
${ }_{12}$ - Hamsadhwani

- Its notes are C, D, E, G, B, C

${ }_{l} D_{13}$ - Dhira Sangarabharanam

- Its notes are C, D, E, F, G, A, B, C

The following attributes of emotions are considered as range space elements of LAFRM.

Emotional Terms considered for the analysis

\begin{tabular}{|c|c|c|}
\hline $\begin{array}{c}{ }_{l} R_{I}-\text { - Наppy } \\
\text { (H) }\end{array}$ & ${ }_{l} R_{2}-\underset{\text { (A) }}{\text { Amazing }}$ & ${ }_{l} R_{3}-$ Love $(\mathrm{L})$ \\
\hline${ }_{l} R_{4}-$ Peace $(\mathbf{P})$ & $\begin{array}{c}{ }_{l} R_{5}-\underset{\text { Nostalgia }}{(\mathrm{N})} \\
\end{array}$ & ${ }_{l} R_{6}-\operatorname{Fear}(\mathrm{F})$ \\
\hline $\begin{array}{c}{ }_{l} R_{7}-\underset{\text { Tension }}{\text { (T) }} \\
\text { (T) }\end{array}$ & ${ }_{l} R_{8}-$ Heroic (B) & ${ }_{l} R_{9}-\operatorname{Sad}(\mathrm{S})$ \\
\hline
\end{tabular}

The group emotion experiences for the songs with different strength of linguistic level matrices are given in the following linguistic level relational matrices.
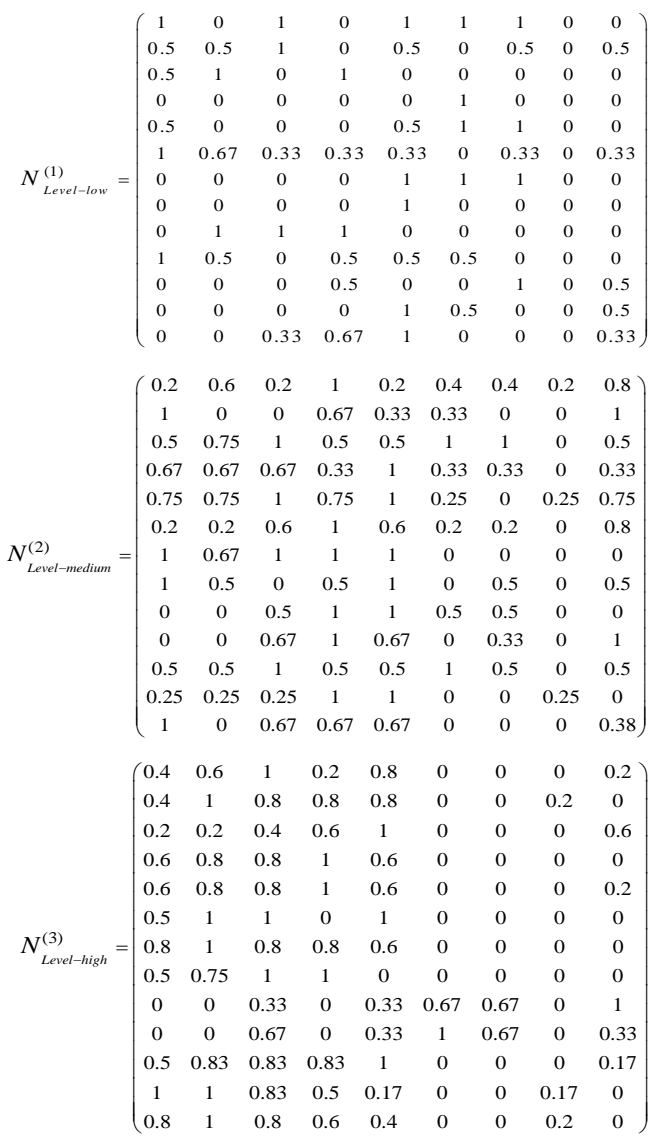

The level matrices are formulated from the table below. The movie and devotional (shaded) songs for survey have been selected from each familiar raga which is given in the Table 1. Their musical features in terms of mode in western notes, rhythm and harmonization are given in the third column. The felt experience levels such as low $(l)$, medium $(m)$, high $(h)$ are given in last column section 
Table 1. Songs and their musical features with experienced emotions level

\begin{tabular}{|c|c|c|c|c|c|c|c|c|c|c|c|}
\hline \multirow{2}{*}{ Raga } & \multirow{2}{*}{ Song } & \multirow{2}{*}{ Musical Features } & \multicolumn{9}{|c|}{ Emotion Level } \\
\hline & & & $\mathbf{H}$ & $\mathbf{A}$ & $\mathbf{L}$ & $\mathbf{P}$ & $\mathbf{N}$ & $\mathbf{F}$ & $\mathbf{T}$ & B & $\mathbf{S}$ \\
\hline \multirow{7}{*}{ 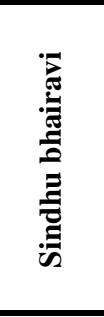 } & [1] Valai osai kala & Em-4/4 - V, F, St - Full & $h$ & $h$ & $h$ & $m$ & $m$ & - & - & - & - \\
\hline & [2] Un thiru yaazhil en & Dm-acc-E-4/4 - Si, St, F & - & $h$ & $h$ & $h$ & $h$ & - & - & - & $h$ \\
\hline & [3] Aasa adhigam vachchi & Dm-6/8 - F, Hack, G & $h$ & $m$ & $h$ & - & - & - & - & - & - \\
\hline & [4] Oru naalum unai & Dm-4/4 - Hack, Tabela, St, F- Full & $l$ & $h$ & $h$ & $m$ & $h$ & $l$ & $l$ & - & $m$ \\
\hline & [5] Ennoda yesuve konjam & Dm-acc-stanza-G\# - 4/4 - Na, Hack, V & - & - & $h$ & $m$ & $h$ & - & - & - & $m$ \\
\hline & [6] Naanoru sindhu & Fm-acc-G-Stan-A-D-B-6/8-V, F - Full & - & $m$ & $m$ & $m$ & $\boldsymbol{h}$ & $m$ & $m$ & - & $m$ \\
\hline & [7] Aattama thearottamaa & Dm - 4/4 - F, Mendalin, S - Rhythm & $m$ & $m$ & $l$ & $m$ & $l$ & $m$ & $m$ & $m$ & $m$ \\
\hline \multirow{6}{*}{ 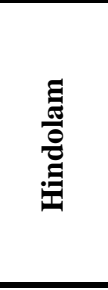 } & [8] Naanaga nanillai & F\#m-acc-stanza-C-A\# - 4/4 - F, Si, G & $m$ & $\boldsymbol{h}$ & $h$ & $h$ & $\boldsymbol{h}$ & - & - & - & $\boldsymbol{h}$ \\
\hline & [9] Maarkazhip poovae & Fm- 4/4 - F-St back & $m$ & $h$ & $h$ & $h$ & $h$ & - & - & - & $m$ \\
\hline & [10] Iraivan enadhu meetpanar & Gm-3/4 - F, Si, St, Tab & $m$ & $\boldsymbol{h}$ & $\boldsymbol{h}$ & $\boldsymbol{h}$ & $\boldsymbol{h}$ & - & - & - & $l$ \\
\hline & [11] Unnaal mudiyum thambi & $\mathrm{Gm}-6 / 8-\mathrm{F}, \mathrm{St}, \mathrm{G}, \mathrm{Tab}-\mathrm{Pu}$. & $h$ & $h$ & $l$ & $m$ & $l$ & - & - & $h$ & - \\
\hline & [12] Vilakku vaippoam & Em - 4/4 - Na, F - Rhythm Change & $l$ & $l$ & $l$ & $m$ & $m$ & - & - & - & $m$ \\
\hline & [13] Pothti vachcha malliga & Bbm-acc- C stanza-A -E-C-A-C - 3/8 & $h$ & $h$ & $h$ & $h$ & $h$ & $m$ & $l$ & - & - \\
\hline \multirow{7}{*}{ 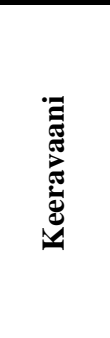 } & [14] Kaatril endhan geedham & Em - 4/4 - F, G, Sax, St, Ha - Pu, Full & $l$ & $m$ & $m$ & $m$ & $m$ & $m$ & $m$ & - & $m$ \\
\hline & [15] Ennai peyar solli & Em - 4/4 - V, St, F, Piano - Full & - & - & $h$ & $h$ & $h$ & - & - & - & $h$ \\
\hline & [16] Nenjikkulle ennavendru & Fm - 6/8 - F, St, Synth, Ha - Full, Pu & $m$ & $m$ & $m$ & $m$ & $m$ & - & - & - & - \\
\hline & [17] Deivame varum ennilae & Dm-acc-C -stanza-C - 7/4 - St, Sax - Ful & $m$ & $m$ & $m$ & $h$ & $h$ & - & - & - & $m$ \\
\hline & [18] Paadi parandha kili & Dm - 6/4-St, F, Na - Full & - & $l$ & $m$ & $l$ & $h$ & $m$ & $m$ & - & $h$ \\
\hline & [19] Deiva tharisanam & $\mathrm{Cm}-6 / 8-\mathrm{F}, \mathrm{Na}, \mathrm{St}-\mathrm{Rhythm}-$ Full, Pu & $h$ & $h$ & $h$ & $h$ & $h$ & $m$ & $m$ & - & - \\
\hline & [20] Engae sellum indha paa & Bm-acc-A - 4/4 - St, F, V - Full & - & $l$ & - & $l$ & $h$ & $m$ & $m$ & - & $h$ \\
\hline \multirow{6}{*}{ 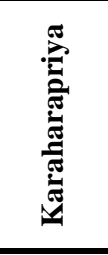 } & [21] Kangal irandaal & Cm-2/4 - F, St, Oboe - Full, Punc. & $h$ & $h$ & $h$ & $h$ & $h$ & - & - & - & - \\
\hline & [22] Arppanam arppanam & Dm-3/4-Si, Na, St, Bell - Punc & - & $m$ & $h$ & $h$ & $h$ & $l$ & - & - & $m$ \\
\hline & [23] Ilankaathtu veesuthe & Dm-4/4 - F, Mand, St, Accor - Full & $h$ & $h$ & $h$ & $h$ & $h$ & - & - & - & - \\
\hline & [24] Thaanaa vandha & Dm-6/8 - G, Syn, Tr, St, F - Full, Pu & $m$ & $m$ & $m$ & $m$ & $m$ & $m$ & $m$ & - & - \\
\hline & [25] Vinnaga virunthe ennil & Cm-6/8 - St, F, Hack - Punc & $\boldsymbol{h}$ & $\boldsymbol{h}$ & $h$ & $h$ & $m$ & - & - & - & - \\
\hline & [26] Aanenna pennenna nee & Dm-acc-stanza-F\# - 6/8 - G,Sax,St - Full & $m$ & $h$ & $m$ & $h$ & $m$ & - & - & - & - \\
\hline \multirow{8}{*}{ 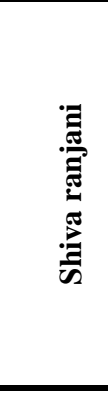 } & [27] Adi aaththaadi & C\#m-acc-stanza-B-F-2/4-F,St,Na,V-Full & $\boldsymbol{h}$ & $\boldsymbol{h}$ & $\boldsymbol{h}$ & $\boldsymbol{h}$ & $m$ & $l$ & $l$ & - & - \\
\hline & [28] Varam kaettu varugindre & Gm-4/4 - Si,St,Bell - Punc & $h$ & $h$ & $h$ & $h$ & $h$ & - & - & - & - \\
\hline & [29] Kaathirunthu kaathirun & Fm-acc-stanza-D\# - A - 6/8 - Na,F- Punc & - & - & $m$ & $h$ & $h$ & $m$ & - & - & $h$ \\
\hline & [30] Endhan idhaya iniya & $\mathrm{Cm}-6 / 8-\mathrm{St}, \mathrm{F}, \mathrm{Si}, \mathrm{Na}-$ Full & $m$ & $h$ & $h$ & $h$ & $h$ & - & - & - & - \\
\hline & [31] Kannum kannum kollai & $\mathrm{Cm}-4 / 4$ - Voice chorus, $\mathrm{G}$ - Full & $h$ & $h$ & $m$ & $m$ & $l$ & - & - & $m$ & - \\
\hline & [32] Maname kalankathiru & Dm-4/4 - Pia, V, F, Sax, St - Full, Pun & $m$ & $m$ & $\boldsymbol{h}$ & $\boldsymbol{h}$ & $m$ & - & - & - & $m$ \\
\hline & [33] Unnai thaanae thanjam & C\#m-acc-F\# stanza-B-F\#-F-6/8-S,F- Full & $m$ & $m$ & $m$ & $m$ & $m$ & $l$ & $l$ & - & $m$ \\
\hline & [34] Innisai paadi varum & Cm-4/4-ac-F-G\#-B- stan-G\#-A\#-B-F-F - Pu & $l$ & $m$ & $m$ & $m$ & $m$ & - & - & - & $m$ \\
\hline \multirow{5}{*}{ 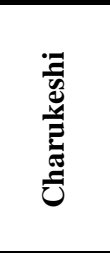 } & [35] Mayankinean solla & E-4/4 - F, Hack, V - Punc & $l$ & $l$ & $h$ & $m$ & $\boldsymbol{h}$ & - & $l$ & - & $m$ \\
\hline & [36] Thoodhu selvathaaradi & A - 4/4 - St, F, Chorus & - & - & $m$ & $h$ & $h$ & $m$ & - & - & $h$ \\
\hline & [37] Thaiyya thaa thaiyya & C-6/8 - F, V, Chorus - Full & $h$ & $h$ & $h$ & $m$ & $h$ & - & - & - & - \\
\hline & [38] Siriya paravai siragai & Am - 4/4-St, F, G, Si - Full & $l$ & $h$ & $m$ & $m$ & $m$ & $m$ & $m$ & - & $m$ \\
\hline & [39] Arumbhagi mottagi & C-4/4-F, St, Syn - Full & $l$ & $l$ & $m$ & $m$ & $m$ & - & - & - & $m$ \\
\hline Raga & Song & Musical Features & $\mathbf{H}$ & $\mathbf{A}$ & $\mathbf{L}$ & $\mathbf{P}$ & $\mathbf{N}$ & $\mathbf{F}$ & $\mathbf{T}$ & B & $\mathbf{S}$ \\
\hline Charu & [40] Aadal kalayae devan & C\# - 4/4 - F, Si, Tabla & $m$ & $m$ & $l$ & $l$ & $l$ & - & - & - & $l$ \\
\hline \multirow{7}{*}{ 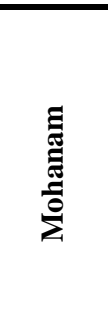 } & [41] Kanmaniyae kaadhal & E-acc- stanza-Bb-D\# -3/4-Si,St,F,Na - Full & $m$ & $m$ & $m$ & $h$ & $m$ & $l$ & - & - & - \\
\hline & [42] Edhu vaendum unakku & D-3/4 - F, St, Hack, Chorus & $h$ & $h$ & $h$ & $h$ & $h$ & - & - & - & - \\
\hline & [43] Ninnukkori varnam & D-4/4 - St, Syn, F - Punc & $m$ & $m$ & $m$ & $m$ & $m$ & - & $l$ & - & - \\
\hline & [44] Iraivan nammai azhai & G\#-6/8 - F, St, Syn, Na - Full & $h$ & $h$ & $h$ & $h$ & $l$ & - & - & - & - \\
\hline & [45] Vellarikkaa pinji & $\mathrm{C} \#-4 / 4-\mathrm{F}, \mathrm{V}$ & $h$ & $h$ & $h$ & $m$ & $m$ & - & - & - & - \\
\hline & [46.a] Iraivanin paliyil inai & C- $2 / 4-\mathrm{St}, \mathrm{Si}$, Chorus, F, G & $m$ & $h$ & $h$ & $h$ & $h$ & - & - & - & - \\
\hline & [46.b] Vaan poalae vannam & D\# -acc- stanza-B - 6/8-F, She, G & $h$ & $h$ & $m$ & $m$ & $h$ & - & - & - & - \\
\hline
\end{tabular}




\begin{tabular}{|c|c|c|c|c|c|c|c|c|c|c|c|}
\hline \multirow{4}{*}{ : } & [47] Iru pookkal - Uyire & E - 4/4 - Rhythm, G - Punc & $m$ & $\boldsymbol{h}$ & $\boldsymbol{h}$ & $m$ & $m$ & - & - & - & $m$ \\
\hline & [48] Pani vizhum malar & $\mathrm{D}-4 / 4-\mathrm{St}, \mathrm{F}, \mathrm{Si}, \mathrm{V}-\mathrm{Punc}$ & $h$ & $h$ & $\boldsymbol{h}$ & $h$ & $l$ & - & - & - & - \\
\hline & [49] Iyangaaru veettu & D-4/4 - Cla, Swaram, Hack, Si & $h$ & $h$ & $\boldsymbol{h}$ & $h$ & $m$ & - & - & - & - \\
\hline & [50] Naru mugaiyae naru & D-acc-stanza-B-A\#-E-B- 4/4-F, Si, St & $m$ & $m$ & $h$ & $h$ & - & - & $m$ & - & - \\
\hline \multirow{3}{*}{ 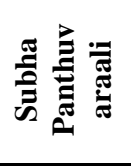 } & [51] Enna enna kanavu & Bm-6/8 - St, F, Pia, Chorus - Full & - & - & $l$ & $m$ & $\boldsymbol{h}$ & $\boldsymbol{h}$ & $h$ & - & $h$ \\
\hline & [52] Vai karayil vaigai kara & Fm-4/4 - Na, St, Si - Full & - & - & $m$ & $l$ & $m$ & $h$ & $h$ & - & $\boldsymbol{h}$ \\
\hline & [53] Unnai naan santhithean & $\begin{array}{l}\text { G\#m-3/4-acc-A\#-C\#-stanza too-F,Chor- } \\
\text { Full }\end{array}$ & - & $l$ & $\boldsymbol{h}$ & $m$ & $m$ & $m$ & $m$ & - & $\boldsymbol{h}$ \\
\hline \multirow{4}{*}{ 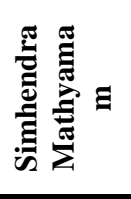 } & [54] Thaalaattum poonthendra & $\mathrm{Cm}-4 / 4-\mathrm{F}$, Hack, St - Full & $l$ & - & $h$ & $m$ & $l$ & $l$ & - & - & $m$ \\
\hline & [55] Aanandha raagam & $\mathrm{Cm}-3 / 4-\mathrm{St}, \mathrm{F}, \mathrm{She}, \mathrm{Na}-$ Full & - & $l$ & $m$ & $m$ & $m$ & $\boldsymbol{h}$ & $\boldsymbol{h}$ & - & $m$ \\
\hline & [56] Idhayamae idhayame un & Bbm-acc-stanza-D\#-6/8 - V,St,Cho - Full & - & - & $m$ & $l$ & $\boldsymbol{h}$ & $\boldsymbol{h}$ & $h$ & - & $\boldsymbol{h}$ \\
\hline & [57] Taji mahal devai illai & $\mathrm{Cm}-4 / 4-\mathrm{St}, \mathrm{V}, \mathrm{F}, \mathrm{Na}-$ Full & $l$ & - & $h$ & $m$ & $m$ & $h$ & $m$ & - & $m$ \\
\hline \multirow{7}{*}{ 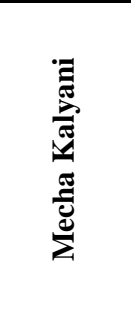 } & [58] Amma endrazhaikkaa & D\#-4/4 - Si, St, F - Punc & - & $\boldsymbol{h}$ & $\boldsymbol{h}$ & $\boldsymbol{h}$ & $\boldsymbol{h}$ & $m$ & - & - & $m$ \\
\hline & [59] Sakthiyanava & $\mathrm{C}-6 / 8-\mathrm{Si}, \mathrm{F}, \mathrm{St}$ & $h$ & $h$ & $m$ & $h$ & $h$ & - & - & - & - \\
\hline & [60] Kaatril varum geethamae & D- 4/4 - St, Hack, F & $m$ & $m$ & $h$ & $h$ & $\boldsymbol{h}$ & - & $l$ & - & $l$ \\
\hline & [61] Aandavarai naan potri & $\mathrm{D}-4 / 4-\mathrm{Si}, \mathrm{St}, \mathrm{F}$ & $h$ & $h$ & $\boldsymbol{h}$ & $h$ & $\boldsymbol{h}$ & - & - & - & - \\
\hline & [62] Vellai puraa ondru & F-acc- stanza-A\#- 4/4-F, Na, She,V- Full & - & - & $h$ & $l$ & $h$ & $m$ & $m$ & - & $h$ \\
\hline & [63] Kalai maangal neer & D- stanza-G-4/4-St, G, Si & $h$ & $h$ & $h$ & $h$ & $h$ & - & - & - & - \\
\hline & [64] Vandhaal mahalakshmi & C\#-4/4 - G, Si, F, Swaram - Punc & $\boldsymbol{h}$ & $\boldsymbol{h}$ & $m$ & $m$ & $m$ & - & $l$ & - & - \\
\hline \multirow{3}{*}{ 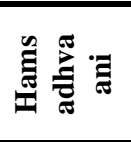 } & [65] Vellai pookkal & E-4/4-G, F & $m$ & $h$ & $l$ & $h$ & $h$ & - & - & - & $l$ \\
\hline & [66] Iraiyesu azhaippaetru & Ok-C-3/4 - Brass, Si, St, Na, F - Punc & $h$ & $h$ & $\boldsymbol{h}$ & $m$ & $l$ & - & - & $h$ & - \\
\hline & [67] Iru vizhiyin vazhiye & 4/4-Syn, St, Bell, Tr, F- Punc & $h$ & $h$ & $h$ & $m$ & $m$ & $l$ & - & $m$ & - \\
\hline \multirow{4}{*}{ 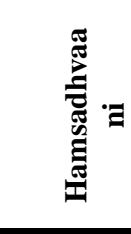 } & [68] Isaiyil swaram searthu & C-4/4 - St, F, Si, Jathi & $\boldsymbol{h}$ & $\boldsymbol{h}$ & $h$ & $m$ & $m$ & - & - & - & - \\
\hline & [69] Vaa vaa vaa kannaa vaa & E-acc-C\#-stanza-A\#-C\#-4/4-Sx, F,St,G - Pu & $h$ & $m$ & $\boldsymbol{h}$ & $m$ & $m$ & - & - & - & - \\
\hline & [70] Tamizhal un pugazh & $\mathrm{D}-4 / 4-\mathrm{Si}, \mathrm{V}, \mathrm{F}, \mathrm{St}$ & $h$ & $h$ & $h$ & $h$ & $l$ & - & - & - & - \\
\hline & [71] Sri ranga ranga naadha & $\begin{array}{l}\text { C\#-acc-A\#-stanza-A\#-4/4-Ha,Si,F,V,St,Na- } \\
\text { F }\end{array}$ & $\boldsymbol{h}$ & $\boldsymbol{h}$ & $m$ & $\boldsymbol{h}$ & $m$ & - & - & - & - \\
\hline \multirow{7}{*}{ 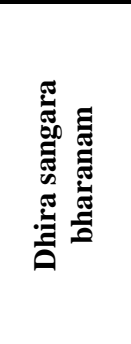 } & [72] Kanmani anbodu kadhala & C\#-acc-stanza-G-A - 6/8 - St, F, Si - Full & $m$ & $h$ & $h$ & $m$ & $h$ & - & - & - & $m$ \\
\hline & [73] Azhaikirar yesu & E-6/8 - St, Brass section, Harmonica - Punc & $h$ & $h$ & $h$ & $h$ & $l$ & - & - & $h$ & - \\
\hline & [74] Anjali anjali anjali min & $\begin{array}{l}\text { D-acc-F-4/4 - Sax, Chorus, St, Hack, F- } \\
\text { Full }\end{array}$ & $\boldsymbol{h}$ & $\boldsymbol{h}$ & $l$ & $l$ & $m$ & - & - & - & - \\
\hline & [75] Ungalukku samadhanam & D-4/4-F, Accor, G, St, Si & $m$ & - & $m$ & $h$ & $m$ & - & - & - & $l$ \\
\hline & [76] Thil paru jaane dhinam & Bb-acc-stanza-C\#-6/8 - She,Hack,F,St - Pu & $m$ & - & $m$ & $m$ & $l$ & - & - & - & - \\
\hline & [77] Kuzhaloodhum kannanu & G-4/4 - F, St, Syn, Hack - Full & $\boldsymbol{h}$ & $\boldsymbol{h}$ & $h$ & $l$ & $l$ & - & - & - & - \\
\hline & [78] Nandriyaal thudhi paadu & E-4/4 - Si, G, St, Synth - Full & $h$ & $h$ & $h$ & $h$ & $h$ & - & - & - & - \\
\hline
\end{tabular}

The combined linguistic relational matrix with the entries $e_{i j}=\max$ linguisticlevel $\left(\max \left(b_{i j}^{(1)}, b_{i j}^{(2)}, b_{i j}^{(3)}\right)\right)$ is given as follows.

$$
M=\left(\begin{array}{ccccccccc}
l & h & h & m & l & l & l & m & m \\
m & h & l & h & h & m & l & h & m \\
m & l & m & l & h & m & m & O & h \\
m & h & h & h & m & l & m & O & m \\
m & h & m & h & m & l & l & m & m \\
l & h & h & m & h & m & l & O & m \\
m & h & m & m & m & l & l & O & O \\
m & h & h & h & m & O & m & O & m \\
O & l & l & m & m & h & h & O & h \\
l & l & h & m & m & h & h & O & m \\
h & h & m & h & h & m & l & O & m \\
h & h & h & m & m & l & O & m & l \\
m & h & h & m & l & O & O & h & m
\end{array}\right)
$$


Using the triangular numbers for the linguistic variables and from aggregation formula given in $\mathbf{2 . 7}$ and $\mathbf{2 . 8}$, the above matrix entries are converted into single numerical value.

$\operatorname{LA}(M)=\left(\begin{array}{ccccccccc}0.267 & 0.733 & 0.733 & 0.5 & 0.267 & 0.267 & 0.267 & 0.5 & 0.5 \\ 0.5 & 0.733 & 0.267 & 0.733 & 0.733 & 0.5 & 0.267 & 0.733 & 0.5 \\ 0.5 & 0.267 & 0.5 & 0.267 & 0.733 & 0.5 & 0.5 & 0 & 0.733 \\ 0.5 & 0.733 & 0.733 & 0.733 & 0.5 & 0.267 & 0.5 & 0 & 0.5 \\ 0.5 & 0.733 & 0.5 & 0.733 & 0.5 & 0.267 & 0.267 & 0.5 & 0.5 \\ 0.267 & 0.733 & 0.733 & 0.5 & 0.733 & 0.5 & 0.267 & 0 & 0.5 \\ 0.5 & 0.733 & 0.5 & 0.5 & 0.5 & 0.267 & 0.267 & 0 & 0 \\ 0.5 & 0.733 & 0.733 & 0.733 & 0.5 & 0 & 0.5 & 0 & 0.5 \\ 0 & 0.267 & 0.267 & 0.5 & 0.5 & 0.733 & 0.733 & 0 & 0.733 \\ 0.267 & 0.267 & 0.733 & 0.5 & 0.5 & 0.733 & 0.733 & 0 & 0.5 \\ 0.733 & 0.733 & 0.5 & 0.733 & 0.733 & 0.5 & 0.267 & 0 & 0.5 \\ 0.733 & 0.733 & 0.733 & 0.5 & 0.5 & 0.267 & 0 & 0.5 & 0.267 \\ 0.5 & 0.733 & 0.733 & 0.5 & 0.267 & 0 & 0 & 0.733 & 0.5\end{array}\right)$

The dynamical system effect on each attribute is calculated with the following procedure. The hidden pattern of the dynamical system $L A(M)$ (relational matrix) for each state vector is to be found. Suppose node ${ }_{l} D_{l}$ is in the $O N$ state and all other nodes are in off state, the causes of the state vector $X_{I}$

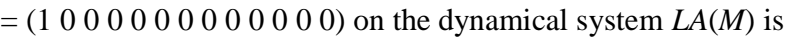
given as follows:

$$
\begin{aligned}
& X_{1} L A(M) \\
& =\left(\begin{array}{lllllllll}
0.267 & 0.733 & 0.733 & 0.5 & 0.267 & 0.267 & 0.267 & 0.5 & 0.5
\end{array}\right) \\
& \hookrightarrow\left(\begin{array}{lllllllll}
0 & 1 & 1 & 0 & 0 & 0 & 0 & 0 & 0
\end{array}\right)=Y_{I} \\
& Y_{1} L A(M)^{T} \\
& =\left(\begin{array}{lllllllll}
1.466 & 1 & 0.767 & 1.466 & 1.233 & 1.466 & 1.233 & 1.466 & 0.534
\end{array}\right. \\
& \begin{array}{llll}
1 & 1.233 & 1.466 & 1.466)
\end{array} \\
& \hookrightarrow\left(\begin{array}{lllllllllllll}
1 & 0 & 0 & 1 & 0 & 1 & 0 & 1 & 0 & 0 & 0 & 1 & 1
\end{array}\right)=X_{2} \\
& X_{2} L A(M) \\
& =\left(\begin{array}{lllllll}
2.767 & 4.398 & 4.398 & 3.466 & 2.767 & 1.301 & 1.534
\end{array}\right. \\
& 1.733 \quad 2.767) \\
& \hookrightarrow\left(\begin{array}{lllllllll}
0 & 1 & 1 & 0 & 0 & 0 & 0 & 0 & 0
\end{array}\right)=Y_{2}\left(=Y_{1}\right) \\
& Y_{2} L A(M)^{T} \\
& =\left(\begin{array}{lllllllll}
1.466 & 1 & 0.767 & 1.466 & 1.233 & 1.466 & 1.233 & 1.466 & 0.534
\end{array}\right. \\
& \begin{array}{llll}
1 & 1.233 & 1.466 & 1.466)
\end{array} \\
& \hookrightarrow\left(\begin{array}{lllllllllllll}
1 & 0 & 0 & 1 & 0 & 1 & 0 & 1 & 0 & 0 & 0 & 1 & 1
\end{array}\right)=X_{3}\left(=X_{2}\right)
\end{aligned}
$$

\begin{tabular}{|c|c|}
\hline Input & Output \\
\hline${ }_{l} D_{l}$ & $\hookrightarrow\left(\begin{array}{l}10 \\
\end{array}\right.$ \\
\hline${ }_{l} D_{2}$ & 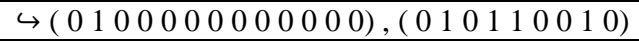 \\
\hline$D_{3}$ & $\hookrightarrow(00010000000000000),(00000100001)$ \\
\hline$D_{4}$ & $\hookrightarrow(0001000100000),(0111100000)$ \\
\hline${ }_{l} D_{5}$ & $\hookrightarrow(0101100100100),(010100000)$ \\
\hline${ }_{l} D_{6}$ & $\hookrightarrow(00000001000000000),(0101010000)$ \\
\hline${ }_{l} D_{7}$ & 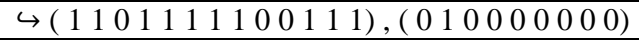 \\
\hline${ }_{l} D_{8}$ & $\hookrightarrow(0000100001000000),(01111000000)$ \\
\hline${ }_{l} D_{9}$ & $\hookrightarrow(0000000010000),(0000011101)$ \\
\hline${ }_{l} D_{10}$ & $\hookrightarrow(00000000000010000),(0010001100)$ \\
\hline${ }_{l} D_{11}$ & $\hookrightarrow(0000000000000100),(11101100000)$ \\
\hline$D_{12}$ & 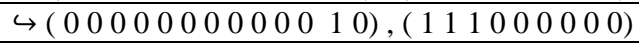 \\
\hline${ }_{l} D_{13}$ & 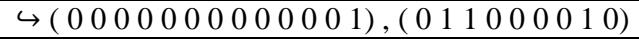 \\
\hline
\end{tabular}

The resultant vector is a fixed point given by the binary pair

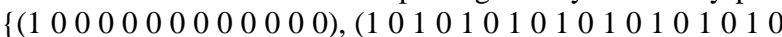

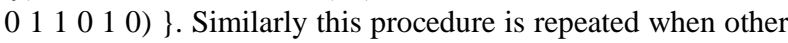
attribute is in $\mathrm{ON}$ state.

Table 2. Fixed Points

\section{CONCLUSION}

The Linguistic Aggregated FRM brings out the factors of emotions for the Carnatic raga songs. The result derived here are the combined levels of experience of a group.

\section{- $\quad$ Sindhu bhairavi}

When this raga songs with $4 / 4$ rhythm of music excerpt $\left({ }_{1} D_{1}\right)$ is in the ON state, amazing and love emotions are felt in high level. Particularly, Devotional songs have got lot of impact in this raga.

\section{- Hindolam}

When this raga scales with $4 / 4,3 / 4$ and $6 / 8$ rhythm of music excerpts $\left({ }_{l} D_{2}\right)$ is in the ON state, people have felt the emotions such as amazing, peace, nostalgia and heroic. This raga gives best results in emotions when it is mingled with accidental notes.

\section{- Keeravani}

When this raga songs with $4 / 4,7 / 4,6 / 8$ rhythm of music excerpts $\left({ }_{l} D_{3}\right)$ is in the ON state, nostalgia and sad emotions are evoked through this raga. These raga based songs are best suitable for bringing devotional feelings with mercy.

\section{Karaharapriya}

When this raga scale with $2 / 4,4 / 4$ and $6 / 8$ rhythm of music excerpts $\left({ }_{1} D_{4}\right)$ is in the ON state, the emotions such as amazing, love and peacefulness are induced through this raga. This is best suitable for love mood in pleasant events.

\section{- Shiva Ranjani}

When the minor scale with $4 / 4$ and $6 / 8$ rhythm of music excerpts $\left({ }_{1} D_{5}\right)$ is in the ON state, The emotions amazing and peace are observed. This raga is suitable when one surrenders themselves to someone with their memories.

\section{- Charukeshi}

When this raga notes with $4 / 4$ and $6 / 8$ rhythm of music excerpts $\left.{ }_{l} D_{6}\right)$ is in the ON state, people have felt the emotions such as amazing, love and nostalgia. The emotion comes as hesitation to show their sentiment feelings.

\section{- Mohanam}

When this raga with $3 / 4,4 / 4$ and $6 / 8$ rhythm of music excerpts $\left({ }_{l} D_{7}\right)$ is in the ON state, amazing emotion was experienced. This implies that love towards God could be expressed in this raga. 


\section{- Chalanattai}

When this raga scales with $4 / 4$ rhythm of music excerpt $\left.{ }_{l} D_{8}\right)$ is in the ON state, the experienced emotions are amazing, love and peace. This raga creates tremendous imaginations in the mind.

\section{- Subha Pandhuvarali}

When this raga notes with any rhythm of music excerpt $\left.{ }_{l} D_{9}\right)$ is in the ON state, the emotions such as fear, tension and sad are evoked in the listener. Moreover this raga brings the feel of sadness and misery.

\section{- $\quad$ Simhendra Madhyamam}

When this raga based music excerpt with $4 / 4,3 / / 4$ and $6 / 8$ rhythm $\left.{ }_{l} D_{10}\right)$ is in the ON state, the listeners mostly felt love, fear and tension emotions. Also these raga songs express sentimental feelings of sadness.

\section{- Mecha Kalyani}

When these raga based any rhythm of music excerpt $\left({ }_{l} D_{11}\right)$ is in the ON state, the emotions amazing, peace, happy and sentiment which are mostly felt by the listeners. Moreover this raga is used for the auspicious events and to praise God.

\section{- Hamsadhwani}

When this raga based 4/4 and 3/4 rhythm of music excerpt $\left.{ }_{(} D_{12}\right)$ is in the ON state, people have felt the emotions such as amazing, love and happy. This raga fills our mind with peace, a sense of praising God and is used mostly during auspicious events.

\section{- Dhira Sangarabharanam}

When this raga based $2 / 4$ rhythm of music excerpt $\left({ }_{1} D_{13}\right)$ is in the ON state, the listeners highly felt the emotions such as amazing, love and heroic. Moreover in comparison with the other ragas this particular raga influences positive thinking.

Thus the combination of Carnatic raga with rhythmic pattern, instrumentation, and harmonization are important for promoting emotions through music.

\section{ACKNOWLEDGEMENT}

This research work is supported by UGC scheme MANF. Award Letter No.: F1-17.1/2011-12/MANF-CHR-TAM7467/(SA-III/Website).

\section{REFERENCES}

[1] Gopala Krishna Koduri, Bipin Indurkhya, "A Behavioral Study of Emotions in South Indian Classical Music and its Implications in Music Recommendation Systems", SAPMIA'10, ACM, Firenze, Italy, October 29, 2010.
[2] Parag Chordia and Alex Rae, "Understanding Emotion in Raag: An Empirical Study of Listener Responses", International Conference on Mathematical Computing (ICMC), Atlanta, 2007.

[3] Patrik N. Juslin \& Daniel Vastfjall, "Emotional responses to music: The need to consider underlying mechanisms", Behavioral and Brain Sciences 31, Cambridge University Press, 2008, p.559-621.

[4] Patra B. G., Das D. \& Bandyopadhyay S., "Automatic Music Mood Classification of Hindi Songs", Proceedings of the $3^{\text {rd }}$ Workshop on Sentiment Analysis where AI meets Psychology, Japan, 2013, p. 24-28.

[5] Richard J. D., Scherer K. R., Hill Goldsmith H, "Handbook of Affective Sciences", Series in Affective Sciences, Oxford University Press, 2003.

[6] Shivani Yardi, Elaine Chew, "Giving Ragas the Time of Day: Linking structure, emotion and performance time in North Indian Classical Music using the Harmonic Network", Proceedings of the 8th International Conference on Music Perception \& Cognition (ICMPC), Evanston, IL, USA, 2004, p.705-708.

[7] Vasantha Kandasamy W.B. and Yasmin,S. "FRM to analyse the Employee-Employer Relationship", Journal of Bihar Mathematical Society, Vol.21, 2001, p. 25-34.

[8] Vasantha Kandasamy W. B., Florentin Smarandache and Ilanthenral, "Elementary Fuzzy Matrix Theory and Fuzzy Models for Social Scientists", Automaton, Printed in the United States of America, 2007.

[9] Victor Devadoss A., Aseervatham S., "Analyze the Effect of Western Classical Music in Chennai using Categorized CETD Matrix" International Journal of Scientific and Engineering Research, Vol.05(08), August, 2014, p. 842-854.

[10] Zadeh L.A., "Fuzzy sets". Information Control 8(3), 1965, p. 338-353.

[11] Zadeh L.A., "The concept of a linguistic variable and its application to approximate reasoning (Part II)", Information Sciences, Vol.08, 1975, p. 301-357.

[12] Zeshui $\mathrm{Xu}$, "Uncertain linguistic aggregation operators based approach to Multiple Attribute Group Decision Making under uncertain linguistic environment". Information Sciences, Vol.168 (01-04), 2004, p. 171184. 